\title{
AN INTERLABORATORY TRIAL TO ASSESS REPEATABILITY AND REPRODUCIBILITY OF MICROBIOLOGICAL PROCEDURES FOR DIAGNOSING INTRAMAMMARY INFECTIONS IN DAIRY COWS
}

\author{
H. Laevens ${ }^{1,2}$, S. De Vliegher ${ }^{1,2}$, L. De Meulemeester ${ }^{3}$, B. Catry ${ }^{1}$, L.A. Devriese ${ }^{4}$, \\ G. Bertels ${ }^{5}$ R. Vandermeersch $^{6}$, E. Mijten $^{7}$, and A. de Kruif ${ }^{1}$ \\ ${ }^{1}$ Department of Reproduction, Obstetrics, and Herd Health, ${ }^{2}$ Veterinary Epidemiology Unit, \\ ${ }^{4}$ Department of Bacteriology, School of Veterinary Medicine, Ghent University, Merelbeke, Belgium; \\ ${ }^{3}$ Animal Health Care Centre, Drongen, Belgium; ${ }^{5}$ Animal Health Care Centre, Alken, Belgium; \\ ${ }^{6}$ Animal Health Care Centre, Torhout, Belgium; 7Animal Health Care Centre, Lier, Belgium.
}

An interlaboratory trial was organised to assess the effect of bacteriological procedures on the repeatability and reproducibility of diagnosing intramammary infections in dairy cows.

Forty milk samples ( 15 were duplicates) of which the majority were from quarters with an elevated somatic cell count (CMT positive) were distributed to 6 laboratories in Flanders (Belgium). Isolation procedure guidelines mentioned a volume of $0.01 \mathrm{ml}$ of milk to be plated on an a-selective blood agar (5\% bovine blood) with a standardised loop. Reading was performed after 24 and 48 hours of incubation at $37^{\circ} \mathrm{C}$. Final results of species identification were reported using the following codes: (1) beta-hemolysin producing staphylococci, (2) other staphylococci, (3) streptococci, enterococci, aerococci, lactococci, (4) Gram negatives (5) Corynebacterium species (6) others (7) contaminant (8) contaminated (more than 2 different species) and (9) negative. Additionally, if 20 or less colony forming units (CFU) were isolated the exact number was reported. Otherwise colony count was reported as ' + '.

Repeatability was evaluated on the 15 duplicate milk samples. Kappa statistic was calculated to assess species identification repeatability and the coefficient of variation was calculated to assess colony count repeatability. Reproducibility was evaluated on the 25 single milk samples. Pairwise kappa statistic was calculated to assess species identification reproducibility. The geometric mean colony count (GMCC) per laboratory was calculated on these samples for which all laboratories reported the same identification code (including negative samples).

Kappa statistic calculated on the 15 duplicate milk samples varied from 0.68 to 0.83 , indicating a good ( $>0.6)$ to very good $(>0.8)$ species identification repeatability. Coefficients of variation varied from 26.7 to $94.0 \%$, indicating poor colony count repeatability. Pairwise kappa statistics between laboratories varied from 0.28 to 0.75 . This large variation could be explained by the large variation of GMCC ( $0.14-1.54 \mathrm{CFU} / 0.01 \mathrm{ml})$. The pairwise kappa statistic was low between laboratories with a large difference in GMCC and high between laboratories with a small difference in GMCC. Also, the smaller the GMCC the higher the proportion of negative results and the larger the GMCC the higher the proportion of samples with 2 or more than 2 different species.

In conclusion, species identification repeatability was good but colony count repeatability and reproducibility were poor. The poor reproducibility could be explained by the differences in GMCC between laboratories. Since the number of CFU is associated with the plating volume, colony count repeatability and reproducibility could be improved by improving standardisation of plating volume procedures. 